\title{
Defective PEX gene products correlate with the protein import, biochemical abnormalities, and phenotypic heterogeneity in peroxisome biogenesis disorders
}

\author{
Nobuyuki Shimozawa, Atsushi Imamura, Zhongyi Zhang, Yasuyuki Suzuki, Tadao Orii, \\ Toshiro Tsukamoto, Takashi Osumi, Yukio Fujiki, Ronald J A Wanders, Guy Besley, \\ Naomi Kondo
}

\begin{abstract}
Department of
Paediatrics, Gifu

University School of

Medicine, 40

Tsukasa-machi, Gifu

500-8076, Japan

N Shimozawa

A Imamura

Z Zhang

Y Suzuki

N Kondo

Faculty of Human Welfare, Chubu

Gakuin University,

Seki 501-3936, Japan

$\mathrm{T}$ Orii
\end{abstract}

Department of Life

Science, Himeji

Institute of Technology,

Kamigori, Hyogo

678-1297, Japan

T Tsukamoto

T Osumi

Department of Biology, Faculty of

Science, Kyushu

University, Fukuoka

812-8581, Japan

Y Fujiki

Department of

Paediatrics and

Clinical Chemistry,

University of

Amsterdam, The

Netherlands

R J A Wanders

Willink Biochemical

Genetics Unit, Royal

Manchester Children's

Hospital, University of

Manchester School of

Medicine, Manchester,

UK

G Besley

Correspondence to:

Dr Shimozawa.

Revised version received 26 April 1999

Accepted for publication

26 May 1999

\begin{abstract}
Peroxisome biogenesis disorders (PBD) comprise three phenotypes including Zellweger syndrome (ZS) (the most severe), neonatal adrenoleucodystrophy, and infantile Refsum disease (IRD) (the most mild), and can be classified into at least 12 genetic complementation groups, which are not predictive of the phenotypes. Several pathogenic genes for PBD groups have been identified, but the relationship between the defective gene products and phenotypic heterogeneity has remained unclear. We identified a mutation in the $P E X 2$ gene in an IRD patient with compound heterozygosity for a missense mutation and the known nonsense mutation detected in ZS patients. In transfection experiments using the peroxisome deficient CHO mutant, Z65 with a nonsense mutation in the $P E X 2$ gene, we noted the E55K mutation had mosaic activities of peroxisomal protein import machinery and residual activities of peroxisomal functions, including dihydroxyacetone phosphate acyltransferase and $\beta$ oxidation of very long chain fatty acids. The nonsense mutation severely affects these peroxisomal functions as well as the protein import. These data suggest that allelic heterogeneity of the $P E X$ gene affects the peroxisomal protein import and functions and regulates the clinical severity in PBD. (F Med Genet 1999;36:779-781)
\end{abstract}

Keywords: Zellweger syndrome; infantile Refsum disease; PEX gene; mosaic

Zellweger syndrome (ZS, McKusick 214100) is the most severe phenotype of peroxisome biogenesis disorders (PBD) and is characterised by the absence of peroxisomes and by multiple metabolic deficiencies, including very long chain fatty acids. Other metabolites accumulate and plasmalogens are deficient. Patients with ZS have a typical face, severe weakness and hypotonia, feeding difficulty, eye abnormalities, and liver dysfunction at birth and death usually occurs within the first year of life. Infantile Refsum disease (IRD, McKusick 266510), the mildest phenotype of PBD, is also characterised by absent peroxisomes and biochemical abnormalities. Patients with IRD show mental retardation, minor facial dysmorphism, and retinitis pigmentosa. Many survive over 10 years old and can walk, as compared with death within a few months in ZS patients, even within the same complementation group. Why IRD patients can survive for over 10 years without peroxisomes has not been elucidated. The milder phenotypes may be because the defective gene products retain partial function. ${ }^{1}$ Nine PEX genes have been identified as pathogenic genes for PBD and almost all of these products are involved in the uptake of peroxisomal matrix proteins. ${ }^{2}$ We report here expression studies of mutated PEX2 genes from patients with ZS and IRD from PBD group $\mathrm{F}$ (group 10 at the Kennedy Krieger Institute), using the PEX2 deficient $\mathrm{CHO}$ mutant. The relationship between the phenotypes, biochemical features, protein import, and gene products at the molecular level was clarified.

Patients F-01 and F-04 had the phenotype of classical ZS and died of heart failure at 8 and 3 months, respectively. Mutation analysis of the $P E X 2$ gene in these patients showed a homozygous mutation of $\mathrm{R} 119 \mathrm{X}$ in $\mathrm{F}-01^{3}$ and a heterozygous mutation of R119X and R125X in F-04. ${ }^{4}$ Patient F-05 was a Welsh female and presented at 1 year with delayed milestones and extreme joint laxity. Later, her retinitis pigmentosa was manifested and biochemical investigations at this stage showed accumulation of very long chain fatty acids and phytanic acid and an abnormal bile acid profile. The activities of dihydroxyacetone phosphate acyltransferase (DHAP-AT) were reduced in platelets and fibroblasts and a diagnosis of infantile Refsum disease was made. She died at 3 years of age following an episode of bronchiolitis. Transfectants with human PEX2 $\mathrm{cDNA}^{3}$ into the F-05 fibroblasts showed peroxisomes (fig 1D), which meant that F-05 was affected by the PEX2 gene deficiency. Mutation analysis showed a heterozygous mutation of R119X and E55K in the PEX2 gene. $^{5}$

Immunofluorescence studies showed no catalase containing particle peroxisome in any of three patients' fibroblasts (fig $1 \mathrm{~B}, \mathrm{C}$ ), in contrast with numerous particles in control fibroblasts (fig 1A). In the study of peroxisomal ghost membrane protein, using anti-human 70 $\mathrm{kDa}$ peroxisomal integral membrane protein (PMP70) antibody, slightly fewer particles were seen in F-05 fibroblasts (fig $1 \mathrm{H}$ ) and 


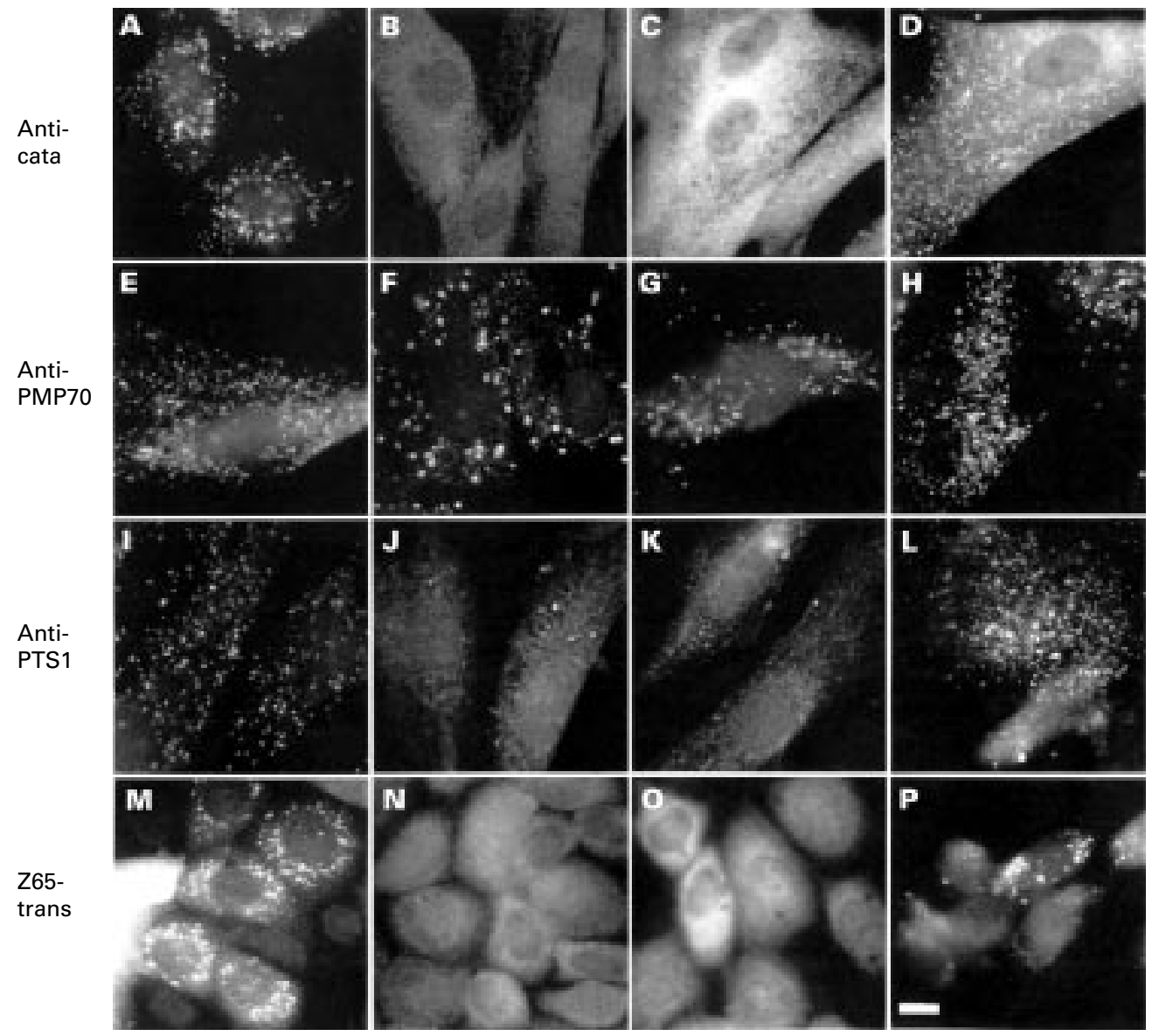

Figure 1 Immunofluorescence staining by antihuman catalase antibody ( $A-D)$, anti-human PMP70 antibody (E-H), anti-PTS1 antibody $(I-L, P)$, and anti-rat catalase antibody $(M-O)$. ( $A, E, I)$ Fibroblasts from control. $(B, F, \mathcal{F})$

Fibroblasts from F-01. (G, K) Fibroblasts from F-04. (C, H, L) Fibroblasts from F-05. (D) F-05 fibroblasts transfected with PEX2-wild cDNA. (M) Z65 transfected with PEX2-wild cDNA. (N) Z65 transfected with PEX2-R125X cDNA. $(O, P) Z 65$ transfected with PEX2-E55K cDNA. Bar=15 $\mu \mathrm{m}$.

larger and still fewer particles were seen in F-01 and F-04 fibroblasts (fig $1 \mathrm{~F}, \mathrm{G}$ ), as compared with findings in control fibroblasts (fig 1E). Further immunofluorescence studies, using anti-peroxisome targeting signal (PTS) 1 antibody, showed hardly any particles in F-01 and F-04 fibroblasts (fig 1J, K), in contrast with numerous particles in control fibroblasts (fig 1I). A somewhat different picture was found in F-05 fibroblasts, in that some cells showed hardly any PTS1 containing particles and others showed numerous particles with PTS1 (fig 1L) (table 1), which means the PTS1 import machinery in F-05 fibroblasts displayed mosaicism.
On biochemical analysis, DHAP-AT activity, a membrane bound peroxisomal enzyme involved in the first step of biogenesis of plasmalogen, was severely diminished in fibroblasts from patients $\mathrm{F}-01$ and F-04 with ZS (6.5\% and $10.6 \%$ of control fibroblasts), determined as described previously. ${ }^{6}$ On the other hand, fibroblasts from F-05 with IRD had residual DHAP-AT activity $(26.1 \%$ of the control) (table 1). Concerning $\beta$ oxidation activity of lignoceric acid relative to that of palmitic acid (C24:0/C16:0), IRD fibroblasts also had residual activity ( $19.2 \%$ of the control), as compared to findings in fibroblasts from patients $\mathrm{F}-01$ and F-04 with $\mathrm{ZS}$ ( $4.4 \%$ and

Table 1 Biochemical and morphological abnormalities which occur with PEX2 gene mutation

\begin{tabular}{|c|c|c|c|c|c|c|c|}
\hline & \multicolumn{2}{|c|}{$D H A P-A T^{\star}$} & \multicolumn{2}{|c|}{$C 24: 0 / C 16: 0$} & \multirow[b]{2}{*}{ Catat } & \multirow[b]{2}{*}{ PMP70† } & \multirow[b]{2}{*}{ PTS1t } \\
\hline & No & $\%$ & No & $\%$ & & & \\
\hline (1) Control & 2.09 & 100 & 0.53 & 100 & +++ & +++ & +++ \\
\hline (2) F-01(ZS; R119X/R119X) & 0.22 & 10.6 & 0.02 & 4.4 & - & + & Rare \\
\hline (3) F-04(ZS; R119X/R125X) & 0.14 & 6.5 & 0.03 & 5.9 & - & + & Rare \\
\hline (4) F-05(IRD;R119X/E55K) & 0.55 & 26.1 & 0.10 & 19.2 & - & ++ & Rare $\sim++\ddagger$ \\
\hline (5) $\mathrm{CHO}-\mathrm{K} 1$ & 2.02 & & 14.3 & & +++ & & \\
\hline (6) Z65 & 0.07 & & 0.92 & & - & & \\
\hline (7) Z65/PEX2-wild & 0.45 & 100 & 8.87 & 100 & ++ & & \\
\hline (8) Z65/PEX2-R125X & 0.10 & 21.9 & 0.56 & 6.3 & - & & \\
\hline (9) Z65/PEX2-E55K & 0.20 & 44.2 & 5.13 & 57.8 & - & & \\
\hline
\end{tabular}

${ }^{\star} \mathrm{nmol} / \mathrm{mg} / 120 \mathrm{~min}$.

HImmunofluorescent punctates with use of anti-human catalase (Cata), anti-PMP70, and anti-PTS1 antibodies (fig 1).

¥Some fibroblasts showed hardly any PTS1 containing particles, others numerous particles with PTS1.

DHAP-AT activities and C24:0/C16:0 are the mean of duplicate experiments. 
$5.9 \%$ of the control), determined as described previously $^{7}$ (table 1).

Next, we investigated the relationship between gene mutation, protein import machinery, and the residual activities using gene transfection. The PEX2-wild, PEX2-R125X, and PEX2-E55K gene sequences subcloned into the expression vector $\mathrm{pUcD} 2 \mathrm{SR} \alpha \mathrm{MCS}^{8}$ were transfected to $P E X 2$ deficient $\mathrm{CHO}$ cell mutants, Z65, ${ }^{9}$ and stable transformants were produced. The transformants of PEX2-wild showed a punctate distribution of catalase, whereas no catalase positive granules were observed in transformants of either PEX2R125X or PEX2-E55K (fig $1 \mathrm{M}-\mathrm{O}$ ). Immunofluorescence studies, using anti-PTS1 antibody, showed a mosaic distribution of PTS1 positive particles in the single colony of Z65 transformants with PEX2-E55K (fig $1 \mathrm{P}$ ), in contrast to no particles in the transformants with PEX2-R125X (data not shown). Biochemical analysis showed that the transformant of $P E X 2-\mathrm{E} 55 \mathrm{~K}$ had residual activities in DHAP-AT $(44.2 \%$ of the PEX2-wild transformant) and C24:0/C16:0 (57.8\% of the PEX2-wild transformant), as compared to the transformant of PEX2-R125X with severely diminished activities, in $21.9 \%$ and $6.3 \%$, respectively, in duplicate experiments (table 1 ). Thus, the E55K mutation of the PEX2 gene retains mosaic activities of peroxisomal import machinery and partial peroxisomal function and regulates the mild phenotype of IRD. On the other hand, the R125X mutation has no peroxisomes functionally and morphologically and regulates the severe phenotype of ZS.

Although there are 12 complementation groups in $\mathrm{PBD},{ }^{10}$ correlation of clinical heterogeneity, biochemical abnormalities, and genetic mutation in groups of PBD was not clear. Our present observations show that the difference between ZS and IRD is the result of different allelic heterogeneity, at least in the $P E X 2$ gene, and this heterogeneity plays an important role in peroxisomal import, determining the phenotypes of $\mathrm{PBD}$, and the biochemical profiles. Even in group $\mathrm{E}$ (group 1 at the Kennedy Krieger Institute) resulting from $P E X 1$ deficiency, there were residual activities of both DHAP-AT and lignoceric acid oxidation in several patients with IRD.
The G843D mutation in PEX1 which was thought to correspond to the IRD phenotype ${ }^{11}$ also had residual activity of lignocelic acid oxidation (data not shown). We have noted that the clinical severity in PBD corresponds to temperature sensitive (TS) mutations, including the E55K mutation in $P E X 2^{5}$ and the TS phenomenon in F-05 may affect her death following infection. Thus, there are several factors which affect the severity and prognosis of PBD, and we are investigating the process by which the defective gene products retaining unstable peroxisomal import machinery result in the clinical heterogeneity and the TS phenomenon.

We thank $\mathrm{K}$ Hori for technical assistance, $\mathrm{M}$ Ohara for assistance with language, and T Imanaka for kindly providing the human PMP70 antibody. This study was supported in part by a Grant-in-Aid for Scientific Research (10670721) from the Ministry of Education, Science, Sports and Culture of Japan.

1 Lazarow PB, Moser HW. Disorders of peroxisome biogenesis. In: Scriver CR, Beaudet AL, Sly WS, Valle D. The metabolic and molecular basis of inherited disease. 7 th ed. New York: A R Liss, 1995:2287-324.

2 Shimozawa N, Suzuki Y, Zhang Z, et al. Nonsense and temperature-sensitive mutations in PEX13 are the cause of temperature-sensitive mutations in PEX13 are the cause of complementation group $\mathrm{H}$ of peroxisom
ders. Hum Mol Genet 1999;8:1077-83.

3 Shimozawa N, Tsukamoto T, Suzuki Y, et al. A human gene responsible for Zellweger syndrome that affects peroxisome assembly. Science 1992;255:1132-4.

4 Shimozawa N, Suzuki Y, Tomatsu S, et al. A novel mutation, $\mathrm{R} 125 \mathrm{X}$ in peroxisome assembly factor-1 responsible for Zellweger syndrome. Hum Mutat 1998;suppl 1:S134-6.

5 Imamura A, Tsukamoto $\mathrm{T}$, Shimozawa $\mathrm{N}$, et al. Temperature-sensitive phenotypes of peroxisome-assembly processes represent the milder forms of human peroxisome-biogenesis disorders. Am f Hum Genet 1998; 62:1539-43.

6 Shimozawa N, Suzuki Y, Orii T, Yokota S, Hashimoto T. Biochemical and morphologic aspects of peroxisomes in the human rectal mucosa: diagnosis of Zellweger syndrome simplified by rectal biopsy. Pediatr Res 1988;24:723-7.

7 Suzuki Y, Shimozawa N, Yajima S, Yamaguchi S, Orii T, Suzuki Y, Shimozawa N, Yajima S, Yamaguchi S, Orii T,
Hashimoto T. Effects of sodium 2-[5-(4Hashimoto T. Effects of sodium 2-[5-(4-
chlorophenyl)pentyl]-oxirane-2-carboxylate (POCA) on fatty acid oxidation in fibroblasts from patients with fatty acid oxidation in fibroblasts from patients with
peroxisomal diseases. Biochem Pharmacol 1991;41:453-6.

peroxisomal diseases. Biochem Pharmacol 1991;41:453-6.
8 Tsukamoto T, Miura S, Nakai T, et al. Peroxisome assembly factor-2, a putative ATPase cloned by functional complementation on a peroxisome-deficient mammalian cell mutant. Nat Genet 1995;11:395-401.

9 Tsukamoto T, Miura S, Fujiki Y. Restoration by a $35 \mathrm{~K}$ membrane protein of peroxisome assembly in a peroxisome-deficient mammalian cell mutant. Nature 1991;350:77-81.

10 Shimozawa N, Suzuki Y, Zhang Z, et al. Genetic basis of peroxisome assembly mutants of humans, CHO cells and yeast: identification of a new complementation group of membrane ghosts. Am f Hum Genet 1998;63:1898-903.

11 Reuber BE, Germain-Lee E, Collins CS, et al. Mutations in PEX1 are the most common cause of peroxisom biogenesis disorders. Nat Genet 1997;17:445-8. 